\title{
Psychometrics Evaluation of the Persian Version of the Swedish Occupational Fatigue Inventory (SOFI), Cultural Adaptation and Reliability
}

\author{
Pashmdarfard M1, Hamid Reza Mokhtarinia ${ }^{2 *}$ and Karimlou $\mathbf{M}^{3}$ \\ ${ }^{1}$ Department of Ergonomics, University of Social Welfare and Rehabilitation \\ Sciences, Iran \\ 2Department of Ergonomics, University of Social Welfare and Rehabilitation Sciences, \\ Iran
}

\section{Research Article \\ Volume 2 Issue 5}

Received Date: August 18, 2018

Published Date: September 17, 2018

DOI: $10.23880 /$ eoij-16000176

${ }^{3} \mathrm{PhD}$ of Biostatistics, Associated professor, Social Determinants of Health research Center, and Biostatistics Department, University of Social Welfare and Rehabilitation Sciences, Iran

*Corresponding author: Hamid Reza Mokhtarinia, Department of Ergonomics, University of Social Welfare and Rehabilitation Sciences, Tehran, Kodakyar Ave. Daneshjo Blvd., Evin, PC: 1985713834, Iran, Tel: +98-21-22180119; Email: hrmokhtarinia@yahoo.com

\section{Abstract}

Introduction: This study assessed the translation, cultural adaptation and reliability of the Persian version of Swedish Occupational Fatigue Inventory Questionnaire (SOFI).

Methods: This cross-sectional study was conducted to evaluate the psychometric characteristics of the SOFI Questionnaire by performing reliability and cultural adaptation. Cultural adaptation was done based on the previous published guidelines and reliability of the SOFI was assessed by internal consistency and test-retest reliability. Testretest reliability was performed in the 50 nurses recorded at baseline and 3-7 days later. Internal consistency was assessed using Cronbach's- $\alpha$.

Results: Some minor changes were applied to the SOFI during the cultural adaptation process. An acceptable level of internal consistency (Cronbach's Alpha= 0.94) and test-retest reliability (ICC=0.9) was obtained.

Conclusion: The Persian version of the SOFI was proved to have sufficient levels of reliability for estimating occupational fatigue of Iranian nurses.

Keywords: Perceived fatigue; Cultural adaptation; Reliability 


\section{Ergonomics International Journal}

Abbreviations: WRF: Work-related fatigue; EMG: Electromyography; EEG: Electroencephalography; EOG: Electrooculography; FMRI: Functional MRI; SOFI: Swedish Occupational Fatigue Inventory; SEM: Standard Error of Measurement; CI: Confidence Interval; ICC: Intra-Class Correlation Coefficient.

\section{Introduction}

The fatigues phenomena is a normal feature that can easily be overcome with sleep and rest in a normal and healthy population [1]. Bills, et al. point out three components for fatigue including, Physiological Fatigue which refers to the decrease in the person's physical ability, objective Fatigue which refers to the decline in the person's performance and efficiency in the system and Subjective Fatigue which refers to the feelings of the fatigue experienced by each person [2].

Work-related fatigues (WRF) are common in the industry and usually are caused by prolonged periods of physical and/or mental exertion without enough time to rest and recovery [3]. Fatigue can compromise health and safety at work and usually results in less productivity [4]. The Side effects of WRF ultimately bring about a serious deterioration in production and productivity in the industry, negatively impacting individuals' motivation, attention, energy, and performance [5,6]. Therefore, the necessity of identifying and determining occupational fatigue in workplace is of paramount importance [7].

Two methods of objective and subjective are used for fatigue evaluation. Objective methods such as electromyography (EMG), electroencephalography(EEG), electrooculography (EOG), functional MRI (FMRI), and PET are expensive and their usability in industry is highly constrained [4,5,8-10]. Self-reported questionnaires are the most common tools which are used as objectives methods The outcome measures are feasible, simple and appropriate method for gathering data about level and quality of fatigue [5]. One of these outcome measures is Swedish Occupational Fatigue Inventory (SOFI), which was designed by Ahsberg in 1998, to assess the physical and mental fatigue in different professions in a single day. One of its priorities in compared to other instruments is ability to report acute occupational fatigue using minimal resources in the least amount of time [8]. Ahsbeg (2000) in another study provided a new short version of SOFI with 20 items and 7-point likert scale. This revised version is widely used [11] and validated in different language such as Spanish [9], China [12].
Due to burgeoning industrialization in Iran, occupational fatigue is a detrimental factor threatening the health of workers in various occupations. It is important to investigate and understand how occupational fatigue is manifested within Iranian industrialization. In order to determine level of fatigue and adopting effective policies for managing it within the labor force, the Persian version of SOFI is necessary. Since the SOFI questionnaire has proved a useful and efficient instrument for evaluating occupational fatigue, its adaptation to the Persian language will be a major and effective step forward in solving this problem. The aims of this study were cross culturally adapt the SOFI for Persian speaking subjects and determine the psychometric properties of reliability and internal consistency in Iranian nurses.

\section{Methods}

\section{Procedure}

Translation process: In the first phase of this study, the permission of translation was adopted from the developer, Mrs. Elizabeth Ahsberg. Translation and crosscultural adaptation of the Persian version of the SOFI was performed based on International published guideline [13] which was fully described in previous study by Shafeai, et al. [14] and Mokhtarinia [15]. Briefly, two native independent Persian translators translated the original English version into Persian and through a session by researchers and translators a consensus version was created. Then it was backward translated to English by another two native English language translators who did not have access to the original English version and familiar with Persian language. The obtained translated English version was sent to the developer and she confirmed the conceptual equivalency of the backward translation with the original version. In the final step, 20 nurses were asked to complete the Persianversion of the SOFI (P-SOFI) and identify any difficult, upsetting or confusing items [16].

Sample: Fifty nurses who were working in a fixed morning shift participated in this study. The average age and work experience were $30.5 \pm 5.5$ and $6.5 \pm 5.12$ years, respectively. The study protocol was approved by the ethics committee of the University of Social Welfare and Rehabilitation Sciences (USWR) and all participants provided written informed consent.

Instruments: SOFI: The short version of the SOFI consisted of 20 items in 5 dimensions (4 items in each dimension), namely: sleepiness, Physical Discomfort, lack of motivation, Lack of energy and Physical exertion, was used in this study [9]. 


\section{Ergonomics International Journal}

\section{Statistics}

To assess the test-retest reliability, the Pr-SOFI was administered to 50 participants with 2 to 3 weeks interval $[10,16,17]$. The time interval between test and retest was selected to diminish the possibility of remembering previous responses [16].

\section{Assessment of Psychometric Properties}

Test-retest reliability was assessed using the two-way random effects model of intra-class correlation coefficient (ICC2, 1) with 95\% confidence interval (CI). An ICC equal to $r>0.70$ is considered acceptable for test-retest reliability. Absolute reliability was assessed by the standard error of measurement (SEM) [18]. Internal consistency was assessed by Cronbach's $\alpha$ coefficient, with an $\alpha$ of 0.70 considered acceptable [19].

\section{Results}

\section{Translation process}

No major modifications were made by forward and backward translators. Because of the lack of equivalent for some phrases in Persian, we select the related and familiar words for some items. For example in Persian we usually categorized pain or fatigue based on its severity from very to less. Item 18 was translated to pain because it was more familiar to Persian people. In item 2 "worn out" was translated to "Exhaustion" in the consensus session.

Also, the results of our pilot study on 20 nurses showed that the items "lack of concern" and "indifference" needed additional explanation for these respondents.

\section{Psychometric Properties}

The results of Cronbach's alpha coefficient for the whole questionnaire as well as its various items are presented in Table1. The highest and the lowest Cronbach's alpha coefficients were 0.94 and 0.88 respectively.

As shown in Table 1, the acceptable level of ICC $\geq 0.70$ was obtained for the total scale and each of the subscales of the SOFI. The ICC obtained for the whole questionnaire was 0.9 , which is indicative of a very high and optimal level of reliability for the Persian version of the SOFI.

SEM was used to compute the absolute reliability, resulting in a value of 3.79 for the whole questionnaire (Table 1).

\begin{tabular}{|c|c|c|c|c|c|}
\hline Subscales & Test (n=50) & Retest (n=50) & ICC(95\% CI) & SEM & Cronbach's $\boldsymbol{~}$ \\
\hline Sleepiness & $14.23(3.11)$ & $12.86(3.48)$ & $\mathbf{0 . 7 8}(0.66-0.87)$ & 1.33 & $\mathbf{0 . 9}$ \\
\hline Physical discomfort & $11.03(6.12)$ & $10.68(4.68)$ & $\mathbf{0 . 8 7}(0.79-0.92)$ & 1.92 & $\mathbf{0 . 9 3}$ \\
\hline Lack of motivation & $7.97(2.67)$ & $9.02(2.86)$ & $\mathbf{0 . 7 7}(0.72-0.90)$ & 1.08 & $\mathbf{0 . 9 1}$ \\
\hline Lack of energy & $14.28(3.26)$ & $16.14(2.95)$ & $\mathbf{0 . 8 6}(0.81-0.93)$ & 0.92 & $\mathbf{0 . 9 4}$ \\
\hline Physical exertion & $7.92(3.11)$ & $7.24(2.93)$ & $\mathbf{0 . 7 9}(0.67-0.88)$ & 1.1 & $\mathbf{0 . 8 8}$ \\
\hline Physical fatigue & $18.33(7.61)$ & $17.92(6.11)$ & $\mathbf{0 . 9}(0.82-0.94)$ & 2.11 & $\mathbf{0 . 9 4}$ \\
\cline { 1 - 4 } $\begin{array}{c}\text { (physical discomfort \& } \\
\text { physical exertion) }\end{array}$ & $22.16(5.34)$ & $21.88(4.74)$ & $\mathbf{0 . 7 2}(0.56-0.83)$ & 2.58 & $\mathbf{0 . 8 4}$ \\
\hline $\begin{array}{c}\text { Mental fatigue (sleepiness } \\
\text { \& lack of motivation) }\end{array}$ & $54.82(13.68)$ & $55.94(11.51)$ & $\mathbf{0 . 9}(0.82-0.94)$ & 3.79 & $\mathbf{0 . 9 4}$ \\
\hline Total SOFI & & & & & \\
\hline
\end{tabular}

Table 1: Test-retest reliability and internal consistency of the Persian SOFI subscales.

Values for test and retest are the mean and SD. ICC and Cronbach's $\boldsymbol{\alpha}$ greater than 0.70 are in bold. Cronbach's $\boldsymbol{\alpha}$ is for the first assessment day.

CI: confidence interval; ICC: intra-class correlation coefficient, SEM: standard error of measurement.

\section{Discussion}

The purpose of the present study was to translate and cross-culturally adapt the original SOFI into Persian and test the Psychometrics properties of the new version. In order to maintain the content validity of an instrument at a conceptual level across different language and cultures, the items must not only be translated well linguistically, but also must be adapted culturally [20-22]. During the translation and cultural adaptation phase, most nurses 


\section{Ergonomics International Journal}

completed the questionnaire unaided, without difficulty and there was no lack of clarity. Some minor modifications in translation were performed for cultural reasons.

In Item 2 and 18, the worn out and ache was translated to Exhaustion and pain because in the Persian language some word have the same meaning and we have not a specifc word for any phrase. For example pain is used for ache and tingling. So we have to use them interchangeable and pain in familiar with Persian people.

The results of this study showed acceptable psychometric properties for use of the Persian SOFI in occupational and research settings. In particular, testretest reliability was 0.9 (95\%CI: 0.83-0.94). The ICC value obtained for the physical fatigue was higher than that of the mental fatigue, which is fairly in line with the results achieved in Leung's study [12]. In this study, similar to the studies on the Chinese and Spanish versions of the SOFI, the obtained value for Cronbach's alpha was greater than $0.9[9,12]$. The SEM value obtained in this study (3.79) was higher than that achieved in Leung's study (0.64), which finding corroborates the finding that the absolute reliability of the Persian version of the SOFI is lower due to the lower sample size in our study compared to Leung's study.

\section{Conclusion}

To our knowledge, the developed Persian version of the SOFI is the only acute fatigue outcome measure in Iran. The results of this study showed it is possible to translate the SOFI questionnaire into Persian without losing the psychometric properties of the original. Consequently, the SOFI-Pr can be applied as a specific fatigue assessment instrument for clinical and research studies in Persian language populations.

\section{Acknowledgment}

This study is part of M.Sc thesis of Mrs. Pashmdar Fard. Special thanks to University of Social Welfare and Rehabilitation Sciences for the financial support. Also, we thank Prof. Ahsberg for permitting us to validate the Persian-version of this instrument in our country, Iran.

\section{References}

1. EB (1956) Fatigue diagnosis and treatment. Ny State J Med 1: 62-67.
2. Gilbert BA (1934) General experimental psychology. Green and co, New York.

3. RS (1953) Motivation in measurements of fatigue in symposium on fatigue. In: Floyd WA (Ed.), Lewis HK \& co, London, pp: 143-148.

4. Winwood PC (2006) Developing and validation of the occupational fatigue exhaustion recovery scale: investigating the significance of non-work time activity in buffering the effects of work strain, in department of psychology. University of South Australia, South Australia.

5. Dallas F (1995) The road to fatigue: circumstance leading to fatigue accidents. In: Laurance $\mathrm{H}$ (Ed.), Taylor \& Francis, London.

6. Mclaugghlin J (2007) Stress, fatigue and workload: determining the combined affect on human performance. In Department of Industrial Engineering and Management Systems, University of Central Florida, California, pp: 345-356.

7. BP (1986) Pathophysiological phenomena in nursing: Human responses to illness. In: Carrier V (Ed.), WB Saners \& co, Philadelphia.

8. Ahsberg E (1998) Perceived fatigue related to work, in department of psychology. University of Stockholm, Swed.

9. Gutiérrez JLG, Bernardo MJ, Eva Garrosa H, Almudena López L (2005) Spanish version of the Swedish Occupational Fatigue Inventory (SOFI): Factorial replication, reliability and validity. International Journal of Industrial Ergonomics 35(8): 737-746.

10. Ghajarzadeh M, Jalilian R, Eskandari G, Ali Sahraian M, Reza Azimi A (2012) Validity and reliability of Persian version of Modified Fatigue Impact Scale (MFIS) questionnaire in Iranian patients with multiple sclerosis. Disabil Rehabil 35(18): 1509-1512.

11. Ahsberg E (2000) Dimentions of fatigue in different working populations. Scand J Psychol 41(3): 231-241.

12. Leung AW, Chan CC, He J (2004) Structural stability and reliability of the Swedish occupational fatigue inventory among Chinese VDT workers. Appl Ergo 35(3): 233-241.

13. Bullinger $\mathrm{M}$, Alonso J, Apolone G, Leplège A, Sullivan $M$, et al. (1998) Translating health status 


\section{Ergonomics International Journal}

questionnaires and evaluating their quality: the IQOLA Project approach. International Quality of Life Assessment. J Clin Epidemiol 51(11): 913-923.

14. Shafeei A, Mokhtarinia HR, Maleki-Ghahfarokhi A, Piri L (2017) Cross-cultural adaptation, validity, and reliability of the persian version of the Orebro Musculoskeletal Pain Screening Questionnaire. Asian spine journal 11(4): 520-530.

15. Mokhtarinia HR, Azadeh Hosseini, Azam MalekiGhahfarokhi, Charles Philip Gabel, Majid Zohrabi (2018) Cross-cultural adaptation, validity, and reliability of the Persian version of the spine functional index. Health and quality of life outcomes 16(1): 1-95.

16. Mazaheri M, Fardipour S, Salavati M, Hadadi M, Negahban H, et al. (2011) The Persian version of Trinity Amputation and Prosthetics Experience Scale: translation, factor structure, reliability and validity, Disabil Rehail 33(19-20): 1737-1745.

17. TDA, NP (2009) Developing and validating a questionnaire to measure spirituality: a psychometric process. Glob J Health Sci 1(1): 11.
18. MD, FP (2000) Quality of life: assessment, analysis and interpretation. In: Chichester (Ed.), John Wiley \& Sons, UK.

19. Bennell KL, Steven Bartam, Kaay Crossley, Sally Green (2000) Outcome measures in patellofemoral pain syndrome: test retest reliability and interrelationship. Int J Sports Phys Ther 1(2): 32-41.

20. Guillemin F, Bombardier C, Beaton D (1993) Crosscultural adaptation of health-related quality of life measures: literature review and proposed guidelines. J Clin Epidemiol 46(12): 1417-1432.

21. Hendricson WD, Russell IJ, Prihoda TJ, Jacobson JM, Rogan A, et al. (1989) Development and initial validation of a dual-language English-Spanish format for the Arthritis Impact Measurement Scales. Arthritis Rheum 32(9): 1153-1159.

22. Guyatt GH (1993) The philosophy of health-related quality of life translation. Qual Life Res 2(6): 461-465. 